\title{
Renal Angiomyolipoma Presenting as Fever of Unknown Origin
}

\author{
Manisha Bhide*, Patricia Bolshoun, Shandra Wilson, Kenneth A. Iczkowski \\ University of Colorado, Denver, USA \\ Email: "Manisha.bhide@Ucdenver.edu
}

Received May 15, 2012; revised June 25, 2012; accepted July 8, 2012

\begin{abstract}
A $50 \mathrm{yr}$ man presented with fatigue and fever. He was found to have a $7.2 \mathrm{~cm}$ left renal mass. Radical nephrectomy was done. The mass was HMB-45 negative renal angiomyolipoma. This patient did not have tuberous sclerosis. His fever resolved after the tumor excision. This case is unique because of the unusual morphology and histology of the tumor. It reiterates that getting rid of inflammatory cytokines being produced by the tumor will cause resolution of fever.
\end{abstract}

Keywords: Fever; Anemia; Elevated ESR; Renal Mass; Angiomyolipoma; Tuberous Sclerosis; Immunohistochemistry

\section{Introduction}

Angiomyolipoma of the kidney is a rare benign tumor that most commonly occurs in association with tuberous sclerosis. Usually it is small and asymptomatic. When large it can present as an abdominal mass with flank pain and hematuria. Here we present a case of fever of unknown origin that was due to renal angiomyolipoma.

\section{Case}

A $50 \mathrm{yr}$ old male nonsmoker presented with fatigue and fever of $101 \mathrm{~F}$ since 1 week. He had well-controlled hypertension. His age-appropriate cancer screening was up to date.

A complete physical exam was normal.

Normochromic normocytic anemia with hemoglobin of $12.2 \mathrm{~g} / \mathrm{dl}$ with significantly elevated erythrocyte sedimentation rate (ESR) and C-reactive protein was noted.

CT scan of abdomen (see Figures 1 and 2) revealed a $7.2 \mathrm{~cm}$ large left upper pole renal mass, which was felt to be renal cell carcinoma. Urologists performed radical left nephrectomy. Lymphovascular and microscopic invasion into perirenal adipose tissue but contained within Gerota's fascia was noted.

Hematoxylin-Eosin stain (Figure 3) showed that the tumor consisted of pleomorphic, mostly round pot-belly cells with plump eosinophilic cytoplasm, macronucleoli, and occasional multinucleate tumor cells. Trabeculae of smooth muscle cells were interspersed through the tumor and contained prominent blood vessels. Mitotic figures were rare. A minority of the tumor area had necrosis and

"Corresponding author. hemorrhage. Thus, by histology it was confirmed to be a pleomorphic epitheloid angiomyolipoma (AML).

An unusual pathological feature was that on immunostaining, unlike $95 \%$ of angiomyolipoma (AML), this tumor was HMB-45 negative. Ours is the first epitheloid AML reported that is negative for pre melanocytic markers including HMB-45, Melan-A and MART (melanoma antigen recognized by $\mathrm{T}$ cells).

The differential diagnosis of renal cell carcinoma was excluded because the tumor cells were positive for calponin and CD68 and negative for pan-cytokeratin.

The patient had an uncomplicated post-operative course. Anemia and fever resolved. The patient is being monitored by Oncologists by 6 monthly surveillance with CT-scan, chest X-ray and complete blood count.

\section{Discussion}

Fever of unknown origin (FUO) is one of the most challenging cases in medicine. The reported incidence of FUO is $2.9 \%$ in hospitalized patients [1].

In developed countries, $20 \%-49 \%$ of cases of fever resolve and cause remains unknown.

The common causes for FUO are connective tissue disease, infection, malignancy or drugs. Malignancy accounts for $7 \%-17 \%$ of causes of fever of unknown origin [1].

The above case illustrates, not only a rare tumor but also reiterates the fact that ablating the source of biological inflammatory cytokines by tumor excision, will dramatically cause regression of fever [2].

A renal mass, which is most commonly associated with fever and accelerated ESR, is renal cell carcinoma [3]. 


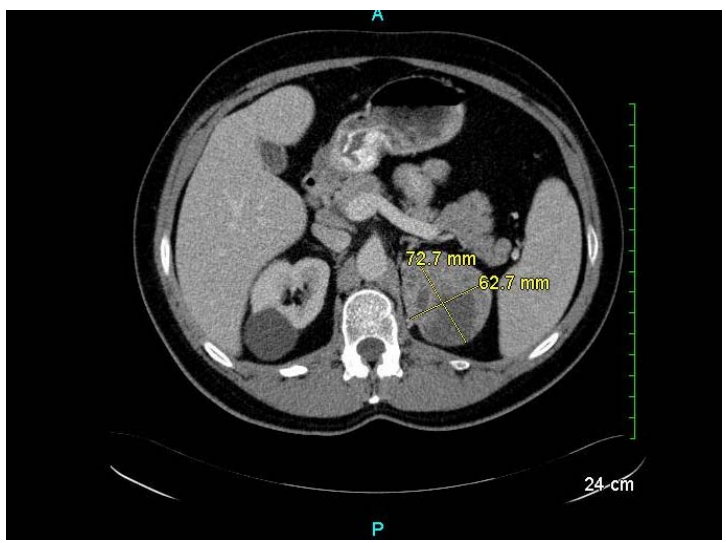

Figure 1. CT scan showing $7.2 \mathrm{~cm}$ solid heterogeneous renal tumor. No enlarged lymph nodes or renal vein invasion.

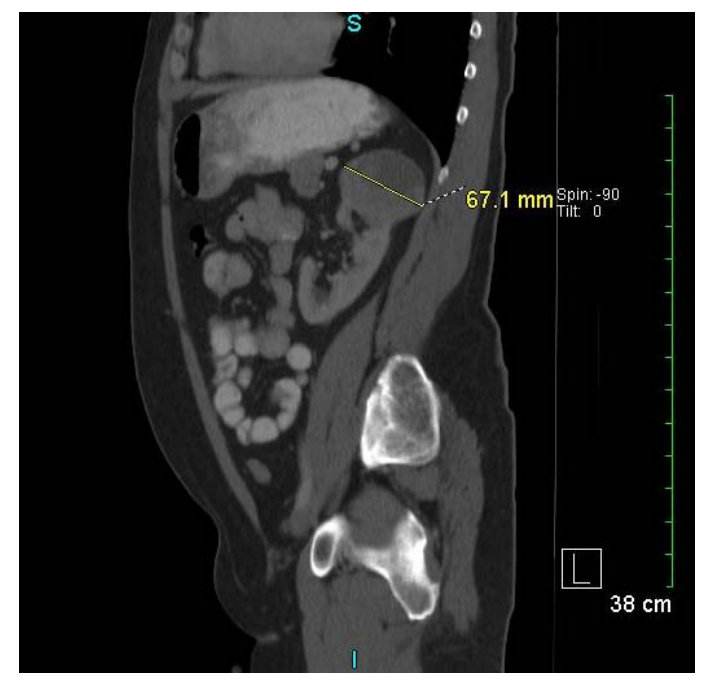

Figure 2. CT of abdomen and pelvis showing left upper pole renal mass extending exophytically superiorly and posteromedially.

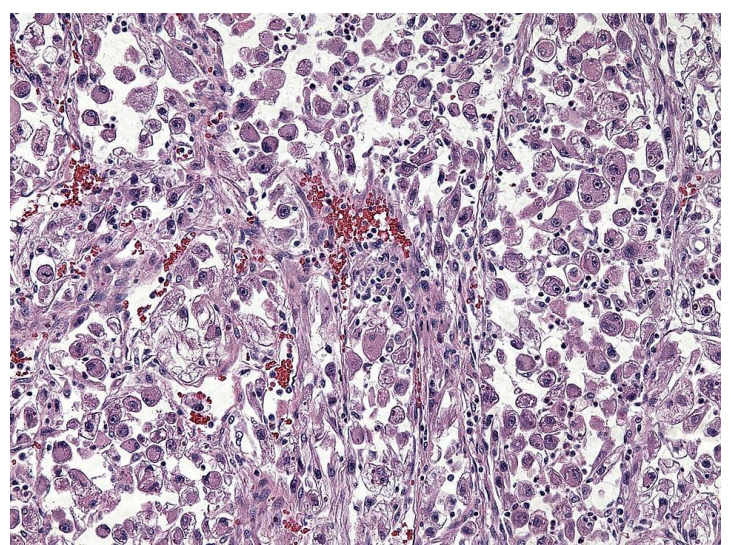

Figure 3. Photomicrograph of angiomyolipoma demonstrating plump, epitheloid cells separated by verticallyoriented strands of smooth muscle cells and blood vessels. A component of fat cells (lipomatous component) is not prominent in this field ( $\mathrm{H}$ and $\mathrm{E}$ stain $20 \times$ objective magnification).
Renal angiomyolipomas are usually benign tumors derived from perivascular epitheloid cells. They contain smooth muscle cells, blood vessels and adipose tissue. They commonly present as an incidental renal mass. The most common serious complication of angiomyolipoma is hemorrhage [4], which occurs in larger tumors [5]. Growing bilateral angiomyolipomas are associated with tuberous sclerosis [6]. Very rarely do they present as a febrile illness.

We could find only 4 cases reported in the literature about a renal angiomyolipoma presenting as FUO that was not associated with tuberous sclerosis [7-10]. Fever can occur with angiomyolipoma alone when associated with tuberous sclerosis. The exact mechanism of this is unknown.

Epitheloid angiomyolipoma is a very rare variant of angiomyolipoma, with only 120 published cases [11]. They are aggressive, potentially malignant mesenchymal tumors, with mean age of presentation around 40 years. Liver, lung and mesentery are the common sites for metastasis. Adverse outcomes were more likely with large size $>7 \mathrm{~cm}$, carcinoma-like growth pattern, renal vein invasion or extra renal spread and when associated with tuberous sclerosis [11].

\section{Conclusion}

Renal angiomyolipoma can be a rare cause for fever of unknown origin. Tumor excision will resolve the fever, but further vigilance with regular follow-up remains important.

\section{REFERENCES}

[1] O. Mourad, et al., "A Comprehensive Evidence-Based Approach to Fever of Unknown Origin," Archives of Internal Medicine, Vol. 163, No. 5, 2003, pp. 545-551. doi:10.1001/archinte.163.5.545

[2] B. Granel, et al., "Difficult Diagnosis of Fever of Unknown Origin Related to an Infraclinic Liposarcoma: Small Tumor and Fever of Unknown Origin," Revue de Médecine Interne, Vol. 24, No. 12, 2003, pp. 819-823.

[3] F. Masuda, et al., "Fever in Renal Cell Carcinoma," Japan Journal of Cancer Clinics, Vol. 31, No. 10, 1985, pp. 1293-1296.

[4] J. N. Eble, et al., "Angiomyolipoma of Kidney," Seminars in Diagnostic Pathology, Vol. 15, No. 1, 1998, pp. 21-40.

[5] C. P. Nelson, et al., "Contemporary Diagnosis and Management of Renal Angiomyolipoma," Journal of Urology, Vol. 168, 2002, p. 1315. doi:10.1097/00005392-200210010-00004

[6] M. S. Steiner, et al., "The Natural History of Renal Angiomyolipoma," Journal of Urology, Vol. 150, No. 6, 1993, p. 1782.

[7] S. Toshiro, et al., "Spontaneous Hemorrhage from Renal 
Angiomyolipoma Presenting with Fever of Unknown Origin," Journal of Internal Medicine, Vol. 46, No. 11, 2007, pp. 793-794.

[8] E. W. Campbell, et al., "Angiomyolipoma Presenting as Fever of Unknown Origin," American Journal of Medicine, Vol. 57, No. 5, 1974, pp. 843-846. doi:10.1016/0002-9343(74)90860-2

[9] S. Sukenik, et al., "Angiomyolipoma Presenting as Fever of Unknown Origin," Israel Journal of Medical Sciences, Vol. 21, No. 10, 1985, pp. 860-861.
[10] K. Vekemans, et al., "Renal Angiomyolipoma as a Cause of Fever of Unknown Origin," British Journal of Urology, Vol. 60, No. 3, 1987, p. 271. doi:10.1111/j.1464-410X.1987.tb05499.x

[11] N. Nese, et al., "Pure Epitheloid PEComas (So-Called Epitheloid Angiomyolipoma) of the Kidney: A Clinicopathologic Study of 41 Cases: Detailed Assessment of Morphology and Risk Stratification," American Journal of Surgical Pathology, Vol. 35, No. 2, 2011, pp. 161-176. doi:10.1097/PAS.0b013e318206f2a9 